\title{
Review of: "Clinical evaluation of non-contact infrared thermometers"
}

\author{
Al Schaefer
}

Potential competing interests: The author(s) declared that no potential competing interests exist.

\section{Review Comment for Scientific Reports}

Dr. Al Schaefer, Dept AFNS, University of Alberta. Edmonton.

Clinical evaluation of non-contact infrared thermometers. 2021. Stacey J. L. Sullivan, Jean E. Rinaldi, Prasanna Hariharan, Jon P. Casamento, Seungchul Baek, Nathanael Seay, OlegVesnovsky \& L.

D.TimmieTopoleski. Scientific Reports. 11: 22079.

I read with interest the recent article published in Scientific Reports. 2021.11:22079 authored by S. Sullivan et al. This is a well conducted study providing an overview of thermal device accuracy, precision and sensitivity for a variety of non contact infrared thermometer (NCIT) devices or often called spot radiometers. The authors are to be commended for their efforts.

This article is particularly timely during the current challenges experienced globally with the covid 19 pandemic. Authorities and health care providers are desperate to try to do something to screen people at risk. This was particularly true before the emergence of rapid antigen tests. Hence, the interest in and use of these thermal devices. Even a cursory look at the current media coverage will demonstrate a plethora of personnel charged with check point duties for screening persons for fever at a host of sites from air ports to sporting venues and commercial businesses. These people can be seen with a variety of mostly hand held devices screening foreheads, cheeks, lateral temporal areas, necks, wrists, arms, eyes, noses and so on. People can also be seen screening for temperatures at different angles to the subject, different distances, under different environmental conditions, through car windows, across car cabins to passengers in the back, and so on and so forth. Hence, not only are there differences in the infrared devices themselves but in the non-standardized manner in which these devices are used. The authors for the aforementioned article appropriately report some of these factors. Hence, it is not surprising that there is variation and confusion in the findings regarding the utility of thermal scanning.

As a thermal physiologist having worked with numerous species of mammals over the past thirty years I would humbly suggest we could augment a few important thermal biology factors to the aforementioned article. Most notably, the issue of timing of the temperature collection and secondly, the issue of thermal symmetry merit discussion.

\section{Timing of Collecting Thermal Data:}

Thermal biometric measurements are some of the most revealing signals of an animal's metabolic state. It has been known since the time of Hippocrates that body temperature measurements are useful indicators 
of health. However, thermal physiology can be complex. Of the five primary mechanisms by which an animal exchanges energy or loses heat to its environment (conductive, convective, radiated, respiratory and evaporative) the radiated heat loss normally represents the primary means of energy exchange. Hence, the utility in choosing radiated heat loss as a proxy for an animal's thermal state. However, it is important to understand the role of radiated heat loss both in normal thermoregulation for a homeothermic animal and particularly during the thermogenesis and pathogenesis of a fever (Schaefer and Cook. 2013. Heat Generation and the role of infrared thermography in pathological conditions. Thermography. Ed by. F. Luzi. P 69-78. Brescia Foundation).

The pathogenesis of a fever in a mammal is nature's defence mechanism designed to harm an invading microbial's thermal preference while allowing the host to tolerate the condition. The generation of a fever involves several phases. Initially in a prodromal phase the animal may well display increased cytokine activity and even serology responses. However, a fever defined as an increase in core temperature will not yet be displayed. The radiated temperature at this stage will be complex. The prostaglandin - hypothalamic response to increase the set point body core temperature may be present. However, the radiated temperature may actually be reduced at this early phase since the animal will be restricting radiated heat loss in an effort to produce the fever. A fever is obtained more through the reduction of heat loss than the increase in heat production. The second and third phases of fever generation will involve an increase in core temperature and a climax core value. During these phases the radiated temperature will also rise and, for a time, be elevated simultaneously with the core temperature. However, once the new temperature hypothalamus set point is reached the corresponding radiated temperature will actually decrease even though the core temperature remains high. Finally, during the fourth phase of a fever the core temperature will also decrease or descend. However, by that time the radiated temperature is already reduced. Hence, it will completely depend on what phase of a fever a person is referring to as to whether there will or will not be a correlation between core and radiated values. Therefore, one must be careful when using radiated temperature as a proxy for core temperature and the timing of these core and radiated temperatures will be absolutely critical to any statistical relationship. Indeed, there will be a period during the pathogenesis of a fever in the second and third phases when radiated and core temperatures will correspond or be correlated. Also, for normal animals in a non-fever state there would be a time sensitive correlation between radiated and core temperatures.

In consideration of the above, regardless of the accuracy of the device, the radiated temperature obtained from the spot radiometers may or may not be a good proxy of core temperature simply due to the biological state of the animal. In this sense, with respect to the aforementioned manuscript, the authors report basically three populations of thermal bins for the people involved in the study; those displaying oral temperatures of 36.1-37.2 C, 37.2 - $38 \mathrm{C}$ and $>38 \mathrm{C}$. There were reportedly some $5 \%$ of the tested subjects with temperatures greater than $38 \mathrm{C}$. In animals, an examination of statistically established cut off values (COV) for known true positive and true negative individuals suggests a radiated temperature value of approximately 37.5 C may be indicative of a viral infection (Schaefer et al Res Vet Sci 2007. 83(3): 376). 
Hence, in the aforementioned study there may be a prevalence of between $5 \%$ and $10 \%$ of the test subjects with an abnormally high temperature. The prevalence of a possible early disease state or true positive state will affect the accuracy of almost any testing technology. The variation in accuracy among the devices tested may be even higher than reported.

\section{Thermal Symmetry:}

Particularly during the early stages of a viral infection it is accurate to say that a change in radiated temperature is associated with the emergence of a viral infection. This has been demonstrated in animals (Schaefer et al Res Vet Sci. 2007. 83(3): 376; Res Vet Sci 2012. 93(2): 928). However, a spot radiometer hand held device such as the devices tested in the aforementioned article may not be sufficient to identify animals at risk. Spot radiometers measure perhaps one or a small number of pixels in the infrared spectrum. Even if these are collected at the correct anatomical site and time, distance, angle, corrected for environmental factors, metabolic factors including variation in stress, time of day, time since eating, state of infection and so forth the fact is the radiometer is only one spot. That may or may not be sufficient to reveal the thermal state of the animal.

Again, with animals (Schaefer and Cook. 2022. Advances in Precision Livestock farming. Burleigh Dodds Sci Pub. In Press), the presence of not just a thermal spot but instead a thermal image profile has been found to be much more revealing. The thermal symmetry of an animal changes with the onset of a pathological condition. With cattle for example diagnosed with either True Positive or Ture Negative clinical conditions for respiratory disease the correlation between the known condition and the infrared thermal profile was significantly greater than with a thermal spot. On the day of clinical identification, the correlation between an infrared thermal spot on a typical cheek skin location was 0.6 compared to the correlation for a thermal profile which included both the cheek skin, the Tmax at the eye and the delta T values between the two $(r=0.94)$. This differentiation was even greater when examined retrospectively three days before the clinical scores validated True Negative and True Positive states (skin cheek $r=0.15$; thermal profile $r=$ 0.85). The data simply suggested that the further evolution of using infrared thermal devices to identify pathological conditions should include the use of thermal imagery and profiles rather than simply a thermal point as is collected currently with these NCIT devices.

In summary, in addition to the importance of the accuracy, precision and sensitivity of a thermal device (NCIT) that the authors professionally report, consideration of the biological state of the animal and the thermal symmetry of the data collected are critical to using radiated temperature information correctly. 